\title{
Index of Refraction Surfaces for Plasma Waves ${ }^{1}$
}

\author{
Tyan Yeh ${ }^{2}$ and Marshall H. Cohen ${ }^{3}$
}

(Received October 15, 1964)

\begin{abstract}
Surfaces of index of refraction have been calculated for the four modes which exist in a warm plasma, ignoring all damping mechanisms. The surfaces are displayed as contour diagrams. Dispersion curves are obtained as profile cuts through the surfaces.
\end{abstract}

\section{Introduction}

The linearized two-fluid plasma theory gives four kinds of waves (four modes); i.e., for every combination of plasma parameters there are four independent plane wave solutions to the linearized set of Maxwell and Euler equations. Many of the properties of these modes have been summarized in various books [e.g., Denisse and Delcroix, 1961; Stix, 1962; and Allis, Buchsbaum, and Bers, 1963]. The new material in this paper consists of details of the surfaces of $n$, the index of refraction, along with techniques for separating the four modes.

The plasma parameters are (subscripts $e$ and $i$ are added later when appropriate)

$m=$ mass of a particle,

$q=$ charge on a particle,

$N=$ particle number density,

$T=$ plasma temperature,

$B=$ static magnetic field strength,

$\theta=$ propagation angle,

$\omega=$ wave frequency.

We shall, however, generally use the following normalized parameters:

$$
\begin{aligned}
& x=\left(\omega_{p}^{2}+\Omega_{p}^{2}\right) / \omega^{2} \\
& y=\omega_{b} \Omega_{b} / \omega^{2} \\
& u=n^{2} \\
& \alpha=c^{2} / V_{e}^{2} \\
& \beta=c^{2} / V_{i}^{2} \\
& \gamma=c^{2} / V_{s}^{2} \\
& K=\cos ^{2} \theta \\
& S=\sin ^{2} \theta
\end{aligned}
$$

${ }^{1}$ Portions of this work were submitted by Tyan Yeh to Cornell University in partial fulfillment of the requirements for the degree M.Sc. in September, 1963.

${ }^{2}$ Department of Applied Mathematics, California Institute of Technology, Pasadena, Calif.

${ }^{3}$ Center for Radiophysics and Space Research, Cornell University, Ithaca, N.Y. 
where

$$
\begin{aligned}
\omega_{p} & =\left(N_{e} q_{e}^{2} / \epsilon_{0} m_{e}\right)^{1 / 2} \\
\Omega_{p} & =\left(N_{i} q_{i}^{2} / \epsilon_{0} m_{i}\right)^{1 / 2} \\
\omega_{b} & =-q_{e} B / m_{e} \\
\Omega_{b} & =q_{i} B / m_{i} \\
V_{e}^{2} & =3 \kappa T / m_{e} \\
V_{i}^{2} & =3 \kappa T / m_{i} \\
V_{s}^{2} & =\left(\omega_{p}^{2} V_{i}^{2}+\Omega_{p}^{2} V_{e}^{2}\right) /\left(\omega_{p}^{2}+\Omega_{p}^{2}\right) \\
c & =\text { velocity of light } \\
\epsilon_{0} & =\text { permittivity of vacuum } \\
\kappa & =\text { Boltzmann's constant. }
\end{aligned}
$$

We shall regard this system of parameters as consisting of the three variables $x, y$, and $u$; and the four independent parameters $Z, r, \alpha, K$ where $Z \equiv q_{i} /\left(-q_{e}\right), r \equiv m_{i} /\left(Z m_{e}\right)$. Note that $\omega_{p}^{2} / \Omega_{p}^{2}$ $=\omega_{b} / \Omega_{b}=r, V_{e}^{2} / V_{i}^{2}=\beta / \alpha=r Z ; \gamma / \alpha=(1+r) /(1+1 / Z)$ and also that $1<\alpha<\gamma<\beta$.

For convenience in comparing this work with that in the books mentioned above, we display some equivalent symbols in the following table:

\begin{tabular}{c|c|c|l}
\hline \hline $\begin{array}{c}\text { Cohen and } \\
\text { Yeh }\end{array}$ & $\begin{array}{c}\text { Denisse and } \\
\text { Delcroix }\end{array}$ & Stix & Allis a et al. \\
\hline$u$ & $y$ & $n^{2}$ & $n^{2}$ \\
$x$ & $x$ & $\alpha$ & $\alpha^{2}$ \\
$y$ & $x / A$ & $\alpha / \gamma$ & $\beta^{2}=\beta_{-} \beta_{+}$ \\
\hline
\end{tabular}

a Minor modifications are introduced when $Z \neq 1$.

The $x$ - $y$ plane is extensively used by Allis et al. [1963] as a background for the CMA diagram (so-named by Stix [1962]), which displays phase-velocity surfaces in 13 characteristic regions of the $x-y$ plane. These surfaces show velocity as a function of $\theta$, and are to be distinguished from the surfaces shown later in this paper, which are contour diagrams of $n^{2}$ for a constant $\theta$. Simplified versions of these contour diagrams have been used in radio astronomy for many years. [Pawsey and Bracewell, 1955.]

\section{Dispersion Equation}

The linearized two-fluid theory of warm plasma gives the dispersion equation as the vanishing of a ninth order determinant. Landau damping does not appear in the theory, even though it is dominant in some régimes. Collision damping may be included, although we do not do so here. The determinant, as given by Denisse and Delcroix [1961], may be expanded to give the dispersion equation in the following form:

$$
A y^{2}-B y+C=0
$$


with

$$
\begin{aligned}
& A=A_{1} x+A_{0} \\
& B=B_{2} x^{2}+B_{1} x+B_{0} \\
& C=C_{3} x^{3}+C_{2} x^{2}+C_{1} x+C_{0}
\end{aligned}
$$

where

$$
\begin{aligned}
A_{1}= & (u-1)(K u-1)(K u / \gamma-1) \\
A_{0}= & (u-1)^{2}(K u / \alpha-1)(K u / \beta-1) \\
B_{2}= & (u-1)(K u / \gamma-1)+(K u-1)(u / \gamma-1) \\
B_{1}= & (u-1)\{(u / \alpha-1)(K u / \beta-1)+(K u / \alpha-1)(u / \beta-1) \\
& +(r+1 / r)(u-1)(K u / \gamma-1) \\
& +S u[(r+1 / r) \\
& \left.\left.+\alpha^{-1}(1-1 / Z)+\beta^{-1}(1-Z)-(1+1 / \gamma)\right]\right\} \\
B_{0}= & (u-1)^{2}[1 / r(u / \alpha-1)(K u / \beta-1)+r(K u / \alpha-1)(u / \beta-1)] \\
C_{3}= & u / \gamma-1 \\
C_{2}= & (u / \alpha-1)(u / \beta-1)+2(u-1)(u / \gamma-1) \\
C_{1}= & (u-1)[2(u / \alpha-1)(u / \beta-1)+(u-1)(u / \gamma-1)] \\
C_{0}= & (u-1)^{2}(u / \alpha-1)(u / \beta-1) ;
\end{aligned}
$$

hence

$$
\begin{aligned}
A= & (u-1)[(K u-1)(K u / \gamma-1) x+(u-1)(K u / \alpha-1)(K u / \beta-1)] \\
B= & K(u-1)[2 x+(\beta / \alpha+\alpha / \beta)(u-1)][(u / \gamma-1) x \\
& +(u / \alpha-1)(u / \beta-1)] \\
& -S(x+u-1)[(u+u / \gamma-2) x \\
& +(u-1)(u / \alpha-\beta / \alpha-u / \beta-\alpha / \beta)] \\
C= & (x+u-1)^{2}[(u / \gamma-1) x+(u / \alpha-1)(u / \beta-1)] .
\end{aligned}
$$

\section{Some Features of the Contours}

Equation (1) is a quartic in $u$. Denisse and Delcroix [1961] have shown that all four values of $u$ are real. In the $x-y$ - $u$ space, (1) is a surface of refractive index, consisting of four sheets, one for each mode. For a given value of $u$, (1) becomes a contour on the $x-y$ plane. We are interested in the structure of the surface of refractive index, but only in the first quadrant.

Some features of the surface can be found readily in analytic form. These are:

(1) The behavior at the origin.

(2) The contour $u=0$.

(3) The contour $u=1$. 
(4) The contours $u= \pm \infty$.

(5) A contour possesses two $x$-axis intercepts (at one, the contour is tangent to the $x$-axis; at the other, it crosses the $x$-axis).

(6) A contour possesses two $y$-axis intercepts (it crosses the $y$-axis at these two intercepts).

(7) A contour possesses two oblique asymptotes.

(8) A contour possesses one vertical asymptote.

(1) At the origin: When $x=0$ and $y=0$, (1) becomes

$$
(u-1)^{2}(u / \alpha-1)(u / \beta-1)
$$

the four values of $u$ are $1,1, \alpha$, and $\beta$.

(2) The contour for $u=0$ (fig. 1): When $u=0$, (1) becomes

$$
(1-x)\left[(y+x-1)^{2}-b y\right]=0,
$$

where $b=\left(r^{1 / 2}-r^{-1 / 2}\right)^{2}$. This contour consists of the line $x=1$ and a parabola whose axis is inclined to the $x$-axis by $3 \pi / 4$ and whose vertex is $(1+3 b / 16, b / 16)$. The parabola is tangent to the $x$-axis at $(1,0)$.

(3) The contour for $u=1$ (fig. 2): When $u=1$, (1) degenerates to

$$
x^{2}[S(1 / \gamma-1) y+(1 / \gamma-1) x+(1 / \alpha-1)(1 / \beta-1)]=0 .
$$

The contour consists of two vertical lines $x=0$, and one oblique line with slope $-1 / S$.

(4) The contour for $u= \pm \infty$ (figs. 3, 4): When $u= \pm \infty$, the coefficient of $u^{4}$ in (1) vanishes, giving

$$
(1 / \alpha \beta)(K y-r)(K y-1 / r)=0 .
$$

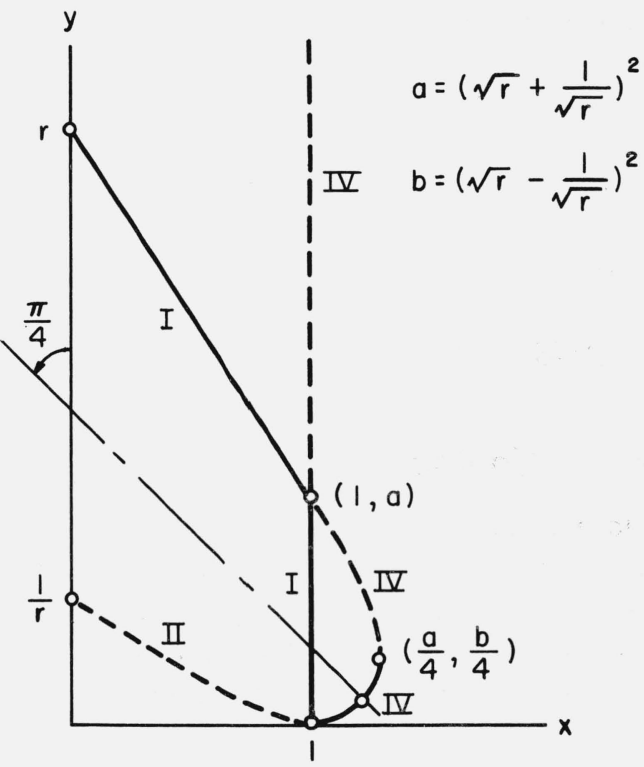

Figure 1. The contour $\mathrm{u}=0$.

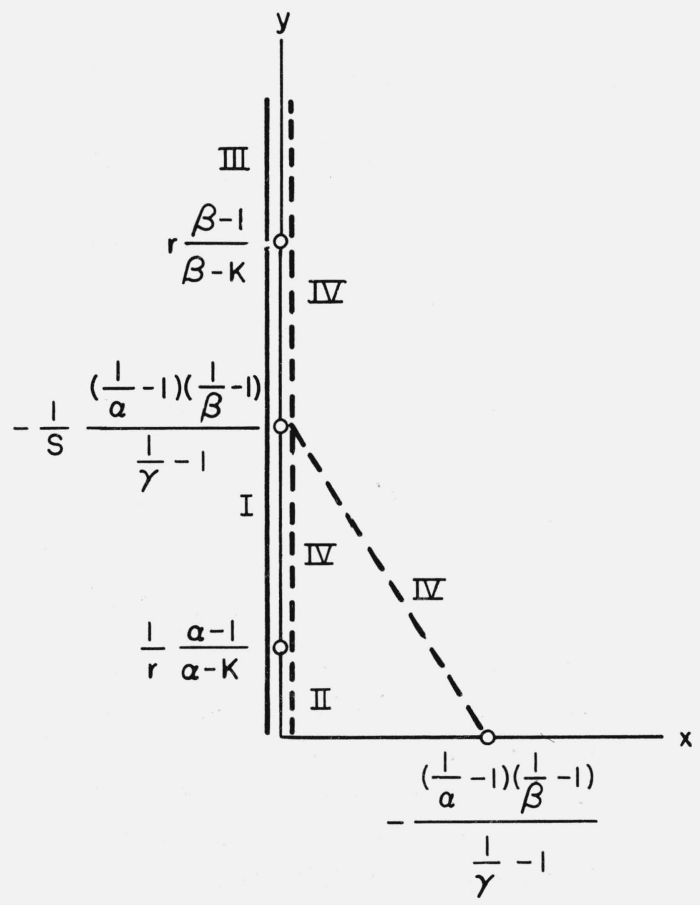

Figure 2. The contour $\mathrm{u}=1$. 


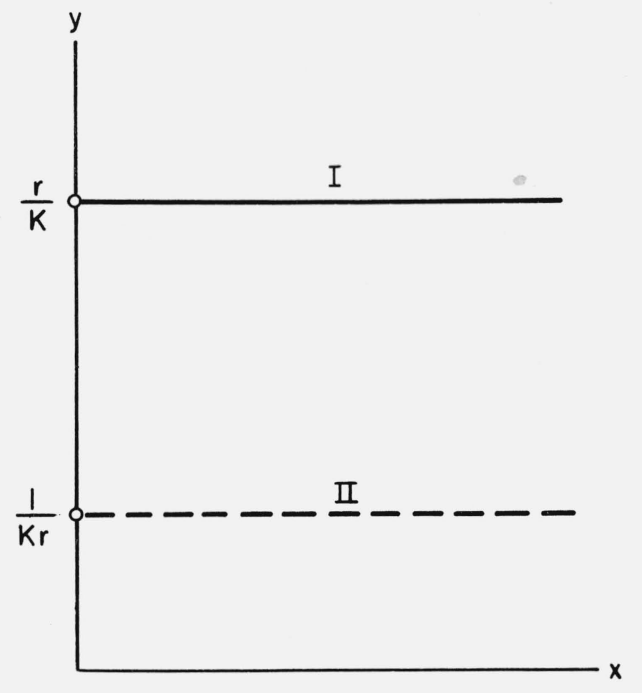

Figure 3. The contour $\mathrm{u}=+\infty$.

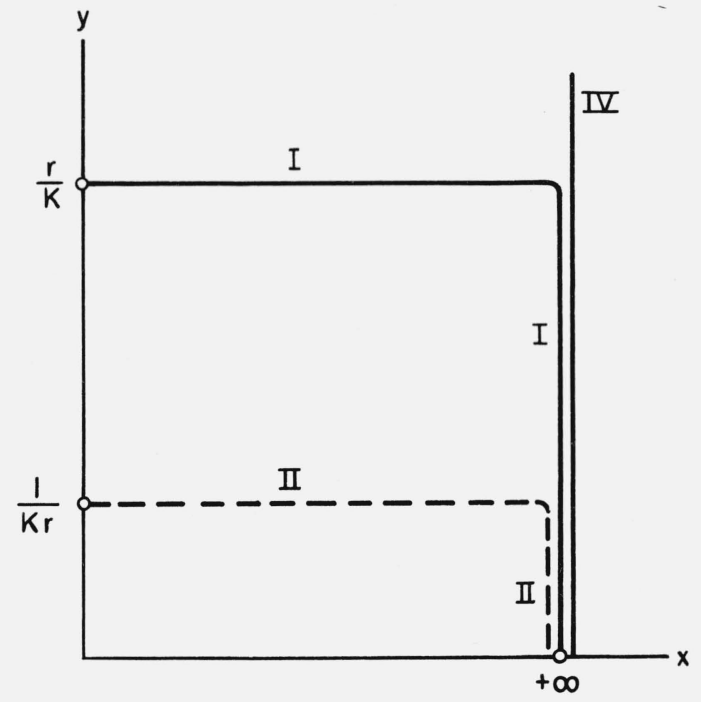

FIGURE 4. The contour $\mathrm{u}=-\infty$.

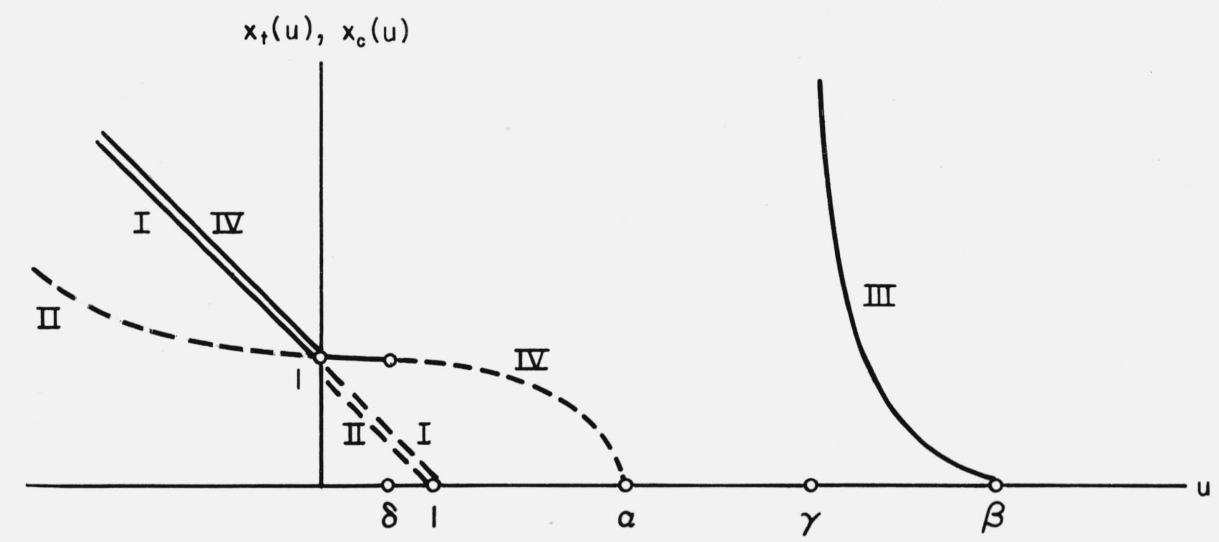

FIGURE 5. Intercepts of the contours on the $\mathrm{x}$-axis, as functions of $\mathrm{u}$.

The contour consists of two horizontal lines $y=r / K$ and $y=1 / K r$. In addition, the contour for $u=-\infty$ has a vertical line at $x=+\infty$ (see (7) below).

(5) The $x$-axis intercepts (fig. 5): When $y=0$, (1) becomes

$$
(x+u-1)^{2}[(u / \gamma-1) x+(u / \alpha-1)(u / \beta-1)]=0 .
$$

At the double zero $x=x_{t}(u)$ where

$$
x_{t}(u) \equiv 1-u
$$


the contour is tangent to the $x$-axis. As the simple zero $x=x_{c}(u)$ where

$$
x_{c}(u) \equiv-(u / \alpha-1)(u / \beta-1) /(u / \gamma-1)
$$

the contour crosses the $x$-axis.

(6) The $y$-axis intercepts (fig. 6): When $x=0$, (1) becomes $(u-1)^{2}\left[(K u / \alpha-1) y-r^{-1}(u / \alpha-1)\right]$ $[(K u / \beta-1) y-r(u / \beta-1)]=0$. At the two zeros $y=y_{a}(u)$ and $y=y_{b}(u)$ where

$$
\begin{aligned}
& y_{a}(u) \equiv r(u / \beta-1) /(K u / \beta-1) \\
& y_{b}(u) \equiv r^{-1}(u / \alpha-1) /(K u / \alpha-1)
\end{aligned}
$$

the contour crosses the $y$-axis. The double zero $u=1$ is part of the contour described in (3) above.

(7) The oblique asymptotes (fig. 7): When $x \rightarrow \infty$ while $y / x$ is fixed,(1) approaches

$$
x[(u-1)(K u / \gamma-1) y-(u / \gamma-1) x][(K u-1) y-x]=0
$$

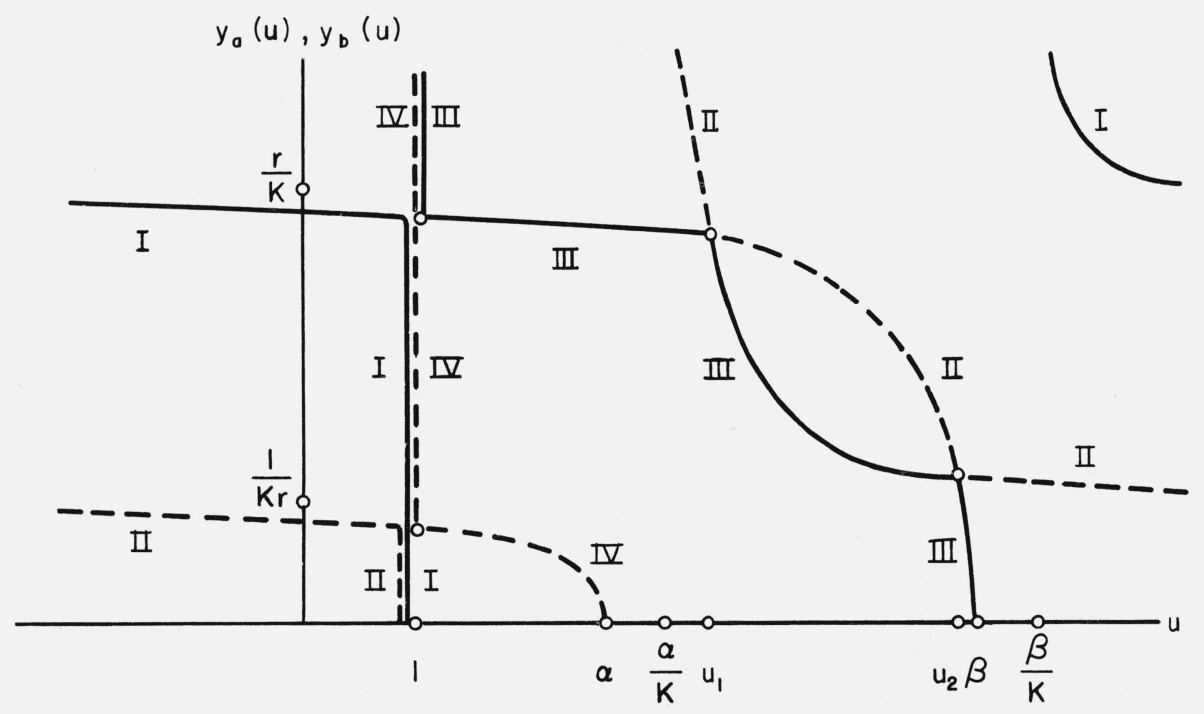

FIGURE 6. Intercepts of the contours on the y-axis, as functions of $\mathrm{u}$.

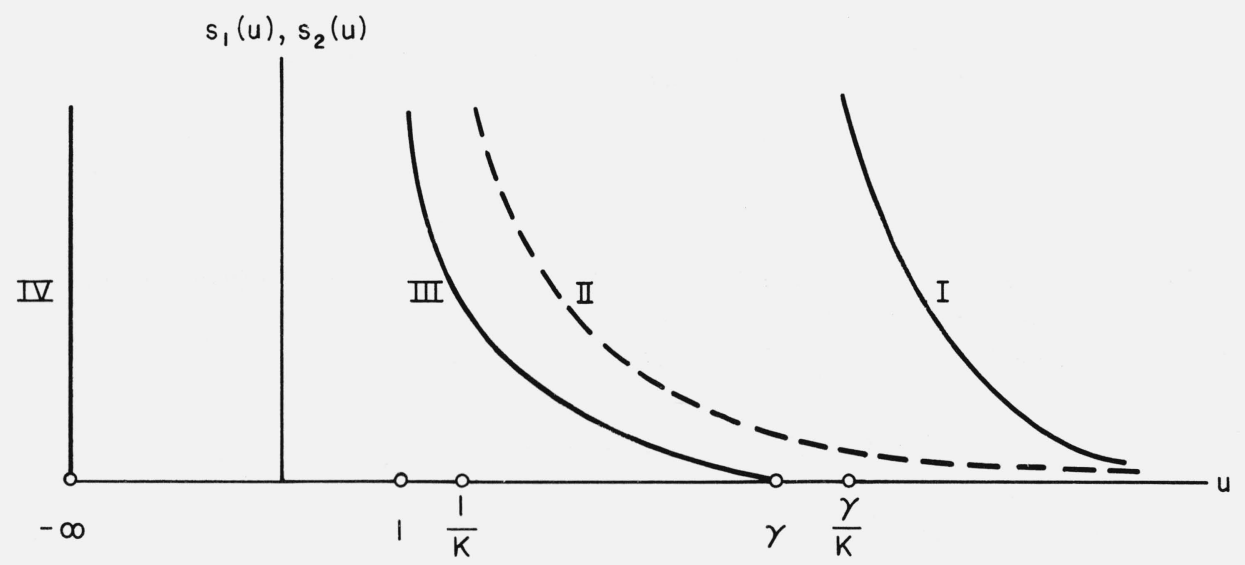

FIGURE 7. Slopes of the oblique asymptotes to the contours, as functions of $\mathrm{u}$. 


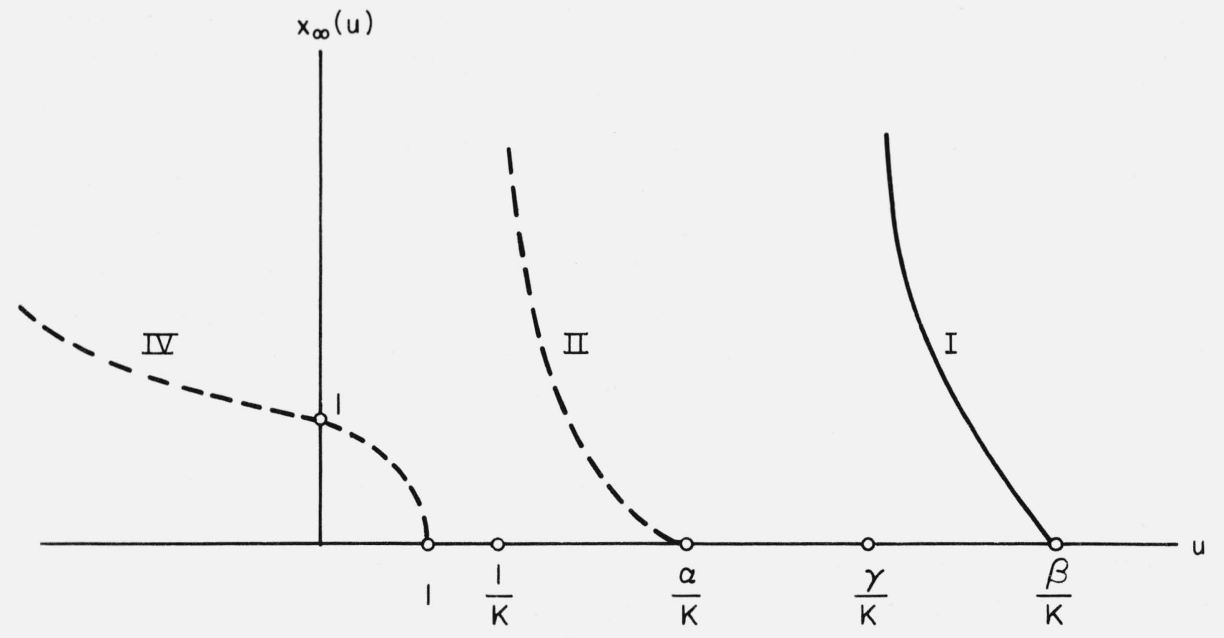

Figure 8. Positions of the vertical asymptotes to the contours, as functions of $\mathrm{u}$.

which gives two oblique asymptotes $y=s_{1}(u) x$ and $y=s_{2}(u) x$ where

$$
\begin{aligned}
& s_{1}(u) \equiv(u / \gamma-1) /(u-1)(K u / \gamma-1) \\
& s_{2}(u) \equiv 1 /(K u-1) .
\end{aligned}
$$

Now (1) is a quartic in $u$, and the product of the four roots is $-\alpha \beta(x-1)\left[(y+x-1)^{2}-\left(r^{1 / 2}-r^{-1 / 2}\right)^{2} y\right] /$ $(K y-r)\left(K y-1 / r\right.$, the ratio of the coefficients of $u^{0}$ and $u^{4}$ in (1). As $x \rightarrow \infty$, one root is $(1+x / y) / K$, given by (7), and the product of two other roots is $\gamma(1+x / y) / K$, given by (6). Hence the fourth root is $-\alpha \beta(x-1) / \gamma$, which shows that there is a contour curve running vertically at $x=+\infty$ when $\boldsymbol{u}=-\infty$.

(8) The vertical asymptote (fig. 8): When $A$ vanishes, which occurs at $x=x_{\infty}(u)$, where

$$
x_{\infty}(u) \equiv-(u-1)(K u / \alpha-1)(K u / \beta-1) /(K u-1)(K u / \gamma-1)
$$

either the root $y_{+}$(if $B>0$ at $x=x_{\infty}$ ) or the other root $y_{-}$(if $B>0$ at $x=x_{\infty}$ ) becomes infinite [see (9) and (10)]. In the vicinity of the vertical asymptote the behavior of the contour is determined by the sign of $A_{1}$.

\section{Contour Curves}

By the contour of a given $u$, we refer to the two branches in the first quadrant of the $x-y$ plane given by $y=y_{+}(x)$ and $y=y_{-}(x)$,

where

$$
y_{+}(x, u) \equiv \frac{B+\sqrt{D}}{2 A}
$$

and

$$
y_{-}(x, u) \equiv \frac{B-\sqrt{D}}{2 A}
$$


where $D=B^{2}-4 A C$. By a contour curve we refer to one continuous section of a contour; it starts at a point on one of the axes, or on an asymptote, and terminates on the other axis, or along an asymptote. Depending on $u$, a contour curve can be given solely by the branch $y=y_{+}(x, u)$, by the branch $y=y_{-}(x, u)$, or by a combination of the two branches joined smoothly at an extreme point $x=x_{e}(u)$ where $D$ vanishes. There can be one, two, three, or four contour curves in a contour.

When $D<0$, the contour does not exist. When $D=0$, the two branches join smoothly at the extreme point. When $D>0$, the two branches are separated. The two branches never cross each other, at most they can be tangent to each other at an extreme point. For in the vicinity of an extreme point, $D$ goes through zero (a) from negative to negative, or (b) from positive to positive, or (c) from one sign to the other. In case (a) the extreme point is an isolated point. In case (b) $y_{+}(x) \geqslant y_{-}(x)$ if $A>0$ at $x=x_{e}$ or $y_{+}(x) \leqslant y_{-}(x)$ if $A<0$ at $x=x_{e}$. The case $A=0$ at $x=x_{e}$ is trivial because then all $A, B$, and $C$ vanish. In case (c) the contour exists only to one side of the extreme point.

The fact that the two branches $y_{+}(x, u)$ and $y_{-}(x, u)$ can not cross each other implies that the four sheets of the refractive index surface do not penetrate into each other. For, each sheet comes either from $y_{+}(x, u)$ or from $y_{-}(x, u)$; if they interpenetrated at some $u=u_{0}$ the corresponding contour curves for $u=u_{0}$ would cross, but they do not.

In the remainder of this section we discuss in detail the special features of section 3 , and identify the contour curves with $y_{+}$and $y_{-}$. In section 4 this identification will be used to help sort the curves into the four modes.

In the figures which follow, the solid curves result from the branch $y=y_{+}(x)$, and the dotted curves result from $y=y_{-}(x)$.

(1) The contour for $u=0$ (fig. 1): When $u=0$,

$$
\begin{aligned}
& A=-(x-1) \\
& B=(x-1)[2 x-(r+1 / r)] \\
& C=-(x-1)^{3} \\
& D=-b(x-1)^{2}(4 x-a)
\end{aligned}
$$

where $a \equiv\left(r^{1 / 2}+r^{-1 / 2}\right)^{2}$ and $b \equiv\left(r^{1 / 2}-r^{-1 / 2}\right)^{2}$. The parabola may be split into two parts: the upper half $y_{1}(x)=-x+4^{-1}(a+b)+2^{-1} \sqrt{b(a-4 x)}$ and the lower half $y_{2}(x)=-x+4^{-1}(a+b)-2^{-1} \sqrt{b(a-4 x)}$. The two halves join at the extreme point $(a / 4, b / 4)$. Since $\sqrt{D}=-(x-1) \sqrt{b(a-4 x)}$ for $x \leqslant 1$ and $\sqrt{D}=(x-1) \sqrt{b(a-4 x)}$ for $1 \leqslant x \leqslant a / 4$, then $y_{+,-}=y_{1,2}(x)$ for $x \leqslant 1$, and $y_{+,-}=y_{2,1}(x)$ for $1 \leqslant x \leqslant a / 4$. The appropriate sections of the parabola in figure 1 are solid and dashed, corresponding to $y_{+}$and $y_{-}$. The vertical line $x=1$ is split up as shown in figure 1 by consideration of the limit $u \rightarrow 0$.

(2) The contour for $u=1$ (fig. 2): As $u \rightarrow 1$, we have, to first order,

$$
\begin{aligned}
A & \rightarrow-S(u-1)(K / \gamma-1) x \\
B & \rightarrow-S(1 / \gamma-1) x^{2} \\
C & \rightarrow x^{2}[(1 / \gamma-1) x+(1 / \alpha-1)(1 / \beta-1)] \\
D & \rightarrow S^{2}(1 / \gamma-1)^{2} x^{4}+4 S(u-1)(K / \gamma-1) x^{3}[(1 / \gamma-1) x+(1 / \alpha-1)(1 / \beta-1)] \\
\sqrt{D} & \rightarrow-S(1 / \gamma-1) x^{2}-2(u-1)(K / \gamma-1) x[x+(1 / \alpha-1)(1 / \beta-) /(1 / \gamma-1)] .
\end{aligned}
$$

Hence $y_{-} \rightarrow-S^{-1}[x+(1 / \alpha-1)(1 / \beta-1) /(1 / \gamma-1)]$ and $y_{+} \rightarrow(1 / \gamma-1) x /(u-1)(K / \gamma-1)$, and the oblique line is given by $y_{-}(x)$, as shown in figure 2 . A consideration of the limit $u \rightarrow 1$ shows that one of the vertical lines is given by $y_{+}$, and the other vertical line is given by $y_{-}$. 
(3) The contour for $u= \pm \infty$ (figs. 3, 4): As $u \rightarrow \pm \infty$,

$$
\begin{aligned}
& A \rightarrow K^{2} u^{4} / \alpha \beta \\
& B \rightarrow(r+1 / r) K u^{4} / \alpha \beta \\
& C \rightarrow u^{4} / \alpha \beta \\
& D \rightarrow(r-1 / r)^{2} K^{2} u^{8} / \alpha^{2} \beta^{2} .
\end{aligned}
$$

Since $\sqrt{D} \rightarrow(r-1 / r) K u^{4} / \alpha \beta$, then $y_{+} \rightarrow r / K$ and $y_{-} \rightarrow 1 / K r$.

(4) The $x$-axis intercepts (fig. 5): When $x=x_{t}(u), B=-K b u(u-1)^{2}[(1 / \gamma-1 / \alpha \beta) u-(1+1 / \gamma)$ $+(1 / \alpha+1 / \beta)]$ which is non-negative if and only if $0<u<(1+1 / \gamma-1 / \alpha-1 / \beta) /(1 / \gamma-1 / \alpha \beta)$. Hence the tangency point results from $y_{+}$when $u<0$, and from $y_{-}$when $0<u<1$. When $x=x_{c}(u)$, $B=-S u[(1 / \gamma-1 / \alpha \beta) u-(1+1 / \gamma-1 / \alpha-1 / \beta)] f(u) /(u / \gamma-1)^{2}$ where $f(u) \equiv(u-1)(u / \gamma-1)\left[\left(r^{-1}\right)(u / \alpha\right.$ $-1)+r(u / \beta-1)]-(u / \alpha-1)(u / \beta-1)(u-1+u / \gamma-1)]$, which has only ${ }^{4}$ one real zero, denoted by $u=\delta$, lying between 0 and 1 . Since $B$ is negative if and only if $0<u<\delta$ or $u>(1+1 / \gamma-1 / \alpha$ $-1 / \beta) /(1 / \gamma-1 / \alpha \beta)$, the crossing point results from $y_{+}(u)$ when $0<u<\delta$ as well as $\gamma<u<\beta$ and from $y_{-}(u)$ when $u<0$ as well as $\delta<\mathrm{u}<\alpha$.

(5) The $y$-axis intercepts (fig. 6): When $x=0$,

$$
\begin{aligned}
& A=(u-1)^{2}(K u / \alpha-1)(K u / \beta-1) \\
& B=(u-1)^{2}\left[r(K u / \alpha-1)(u / \beta-1)+r^{-1}(u / \alpha-1)(K u / \beta-1)\right] \\
& C=(u-1)^{2}(u / \alpha-1)(u / \beta-1) \\
& D=(u-1)^{4}[g(u)]^{2}
\end{aligned}
$$

where $g(u) \equiv r(K u / \alpha-1)(u / \beta-1)-r^{-1}(u / \alpha-1)(K u / \beta-1)$. The discriminator of $g(u)$ may be written

$$
(\beta / Z-\alpha Z)^{-2}\left\{(r-1 / r)^{2}-[\sqrt{r Z}-1 / \sqrt{r Z}]^{2}\right\}^{2}\left(K-K_{1}\right)\left(K-K_{2}\right),
$$

which is negative if and only if $K$ is in the very small range $K_{1}<K<K_{2}$, where

$$
\begin{aligned}
& K_{1}=(\sqrt{r / Z}-\sqrt{Z / r})^{2} /(r-1 / r+\sqrt{r Z}-1 / \sqrt{r Z})^{2} \\
& K_{2}=(\sqrt{r / Z}-\sqrt{Z / r})^{2} /(r-1 / r-\sqrt{r Z}+1 / \sqrt{r Z})^{2} .
\end{aligned}
$$

If this inequality holds, $g(u)$ is positive-definite and the two curves $y_{a}(u)$ and $y_{b}(u)$ [in (4) and (5)] do not intersect. Since $\sqrt{D}=(u-1)^{2} g(u), y_{+}=y_{a}(u)$ and $y_{-}=y_{b}(u)$. Otherwise, $g(u)$ is negative if and only if $u_{1}<u<u_{2}$ where $u_{1}$ and $u_{2}$ are the two real zeros of $g(u)$, lying between $\alpha / K$ and $\beta$. The two curves $y_{a}(u)$ and $y_{b}(u)$ intersect at $u_{1}$ and $u_{2}$, and $y_{+}$and $y_{-}$switch between $y_{a}(u)$ and $y_{b}(u)$ at $u_{1}$ and $u_{2}$. Thus, for $u_{1} \leq u<u_{2}, \sqrt{D}=-(u-1)^{2} g(u)$. Hence $y_{+}=y_{b}(u)$ and $y_{-}=y_{a}(u)$. For $u<u_{1}$ as well as $u>u_{2}, \sqrt{D}=(u-1)^{2} g(u)$, hence $y_{+}=y_{a}(u)$ and $y_{-}=y_{b}(u)$.

Figure 6 is drawn for the case $K_{2}<K \leqslant 1$; i.e., $\theta$ not too close to $90^{\circ}$. The curve $y=y_{a}(u)$ and the line $u=1$ intersect at the point $y=r(\beta-1)(\beta-K)$. The curve $y=y_{b}(u)$ and the line $u=1$ intersect at the point $y=r^{-1}(\alpha-1)(\alpha-K)$.

${ }^{4}$ This point has been established by numerical calculations. 
When $K=K_{2}$, where $\alpha / K<u_{1}=u_{2}<\beta$, the two curves are tangent at a point in the first quadrant. When $K_{1}<K<K_{2}$ the two curves are separate. When $K=K_{1}$, where $\beta<u_{1}=u_{2}$ $<\alpha / K$, the curves are tangent in the fourth quadrant. When $0<K<K_{1}$ the curves intersect in the fourth quadrant. Since only positive $y$ has physical significance, we can state that the curves switch between $y_{+}$and $y_{-}$unless $K<K_{2}$, or, approximately, $\theta>\cos ^{-1}\left(m_{e} / m_{i}\right)^{1 / 2}$.

(6) The oblique asymptotes (fig. 7): As $x \rightarrow \infty$ while $y / x$ is fixed,

$$
\begin{aligned}
& A \rightarrow(u-1)(K u-1)(K u / \gamma-1) x \\
& B \rightarrow[(u-1)(K u / \gamma-1)+(K u-1)(u / \gamma-1)] x^{2} \\
& C \rightarrow(u / \gamma-1) x^{3} \\
& D \rightarrow[h(u)]^{2} x^{4}
\end{aligned}
$$

where $h(u) \equiv(K u-1)(u / \gamma-1)-(u-1)(K u / \gamma-1)=S u(1-1 / \gamma)$. For $u \geqslant 0, h(u)$ is nonnegative, and $\sqrt{D}=h(u), y_{+}(x, u)=s_{1}(u) x$, and $y_{-}(x, u)=s_{2}(u) x$.

(7) At the origin: When $u=\alpha$ and $x=0, A=-S(\alpha-1)^{2}(K \alpha / \beta-1), B=S(\alpha-1)^{2}(1-\alpha / \beta) r$, $C=0, \sqrt{D}=S(\alpha-1)^{2}(1-\beta / \alpha) r$; hence $y_{-}=0$. When $u=\beta$ and $x=0, A=-S(\beta-1)^{2}(K \beta / \alpha-1)$, $B=-S(\beta-1)^{2}(\beta / \alpha-1) / r, C=0, \sqrt{D}=S(\beta-1)^{2}(\beta / \alpha-1) / r$; hence $y_{+}=0$.

(8) A further special case which will be used later is the calculation of $y_{+}$when $u=\gamma$ and $x \rightarrow+\infty$ : In this case $A \rightarrow-S(\gamma-1)(K \gamma-1) x, B \rightarrow-S(\gamma-1) x^{2}, C \rightarrow(\gamma / \alpha-1)(\gamma / \beta-1) x^{2}$, $\sqrt{D} \rightarrow-B+2 A C / B$; hence $y_{+} \rightarrow C / B=-(\gamma / \alpha-1)(\gamma / \beta-1) / S(\gamma-1)$.

\section{Separation of Modes}

The foregoing analyses allow us now to separate the four modes; that is, to identify contour curves for various values of $u$ with one of the four sheets of the index of refraction surface. This separation can be done in an unambiguous way because the four sheets do not penetrate into each other, and the contour curves do not cross, as shown in section 4 .

Consider figures 5, 6, and 7. Each is a profile of the index surface, made by cutting the surface by the planes $y=0, x=0$, and $x=+\infty$, respectively. The curves labeled I, II, III, IV must each belong to one mode, else (in figs. 5 and 6 ) the curves would cross. We conjecture that the introduction of collisions would remove the points of contact and would leave the curves entirely distinct as they already are in figure 7 .

Curves 5III and 6III have a common point $(x, y, u)=(0,0, \beta)$. They both come from $y_{+}(x, u)$ and hence are different profiles of the same sheet of the index surface; they belong to the same mode. Curve 5III is symptotic to the point $[+\infty,-(\gamma / a-1)(\gamma / \beta-1) / S(\gamma-1)$, $\gamma]$, which corresponds to the point $(0, \gamma)$ on curve 7III. Curves 5 III and 7III both come from $y_{+}(x, u)$, and these belong to the same mode. The three curves 5III, 6III, and 7III therefore all belong to one mode, call it mode III. By the principle of nonpenetration, the three curves 5IV, 6IV, and 7IV, immediately to the left of 5III, 6III, and 7III, must be profiles of one mode; call it mode IV. Similarly, the curves labeled I and II must belong to the other two modes.

Any contour curve at least has a point on the $x$-axis or the $y$-axis, or is asymptotic to an oblique asymptote. From the identification of these features with modes according to figures 5,6 , and 7 , the mode to which a contour belongs is uniquely determined. Ambiguities which arise at one end of a contour (e.g., the contour which has a $y$-axis intercept at the point of contact between curves $6 \mathrm{II}$ and 6III) are resolved at the other end of the contour.

In this fashion we can construct the four index surfaces in a unique way. If we look from above towards the origin, we see (in the first octant) the sheets in the sequence I, II, III, IV, then two further sheets of modes I and II. This nomenclature agrees with that of Denisse and Delcroix [1961]. This method of separation is valid for any propagation angle. The details of the surface change with $\theta$, however, especially as $\theta \rightarrow 90^{\circ}$. 


\section{Index of Refraction Surfaces}

Figures 9, 10, 11, and 12 show, in a schematic fashion, the index surfaces for the four modes; the solid and dashed portions of the curves correspond to $y_{+}(x, u)$ and $y_{-}(x, u)$, respectively.

Figures 13, 14, 15, and 16 show the detailed surfaces for the case $r=100, Z=1, \alpha=50, \theta=30^{\circ}$. This is not a realistic plasma, but this choice of parameters does display the various features of the surfaces in a convenient manner. The contour curves are labeled with their values of $u$. The calculations are made from (1) by choosing sets of values of $u$ and $x$ and solving for $y$ on a digital computer. The resulting curves are sorted into modes by examining endpoints and asymptotes, as described in the preceding section.

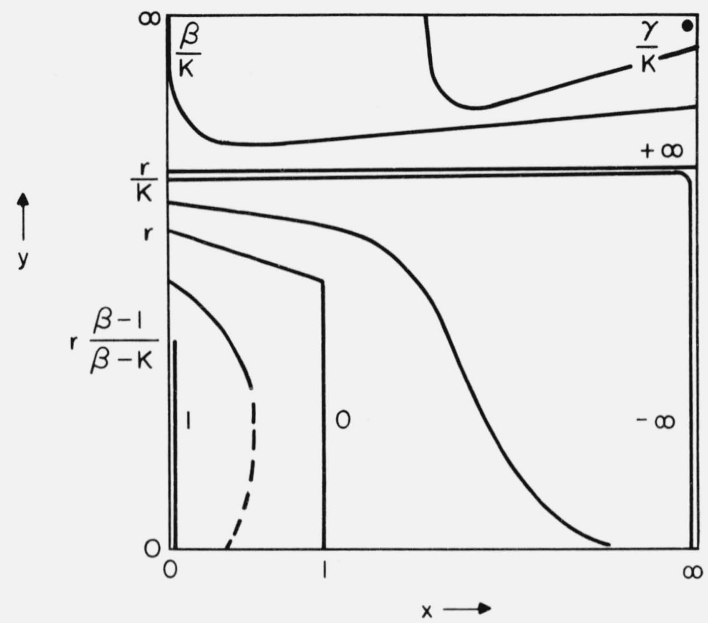

FIGURE 9. Schematic contour diagram of index of refraction for mode $\mathrm{I}$, on $\mathrm{x}-\mathrm{y}$ plane.

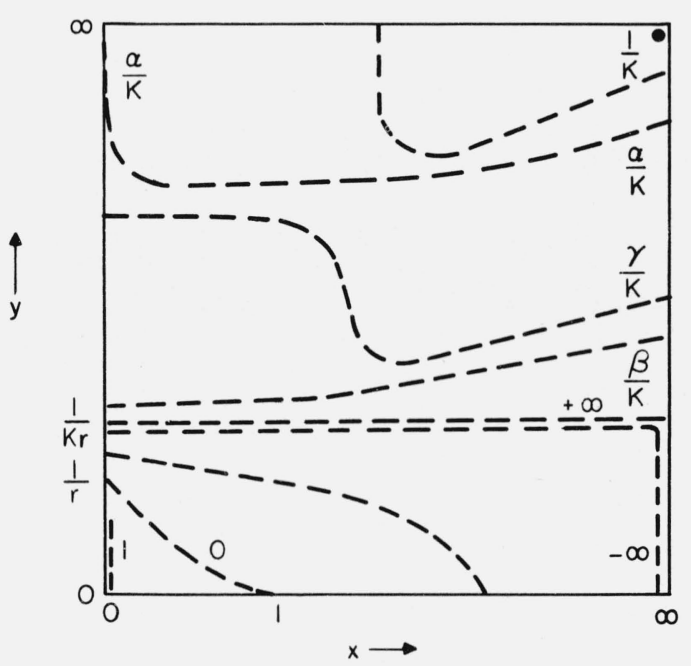

Figure 10. Schematic contour diagram of index of refraction for mode II, on x-y plane.

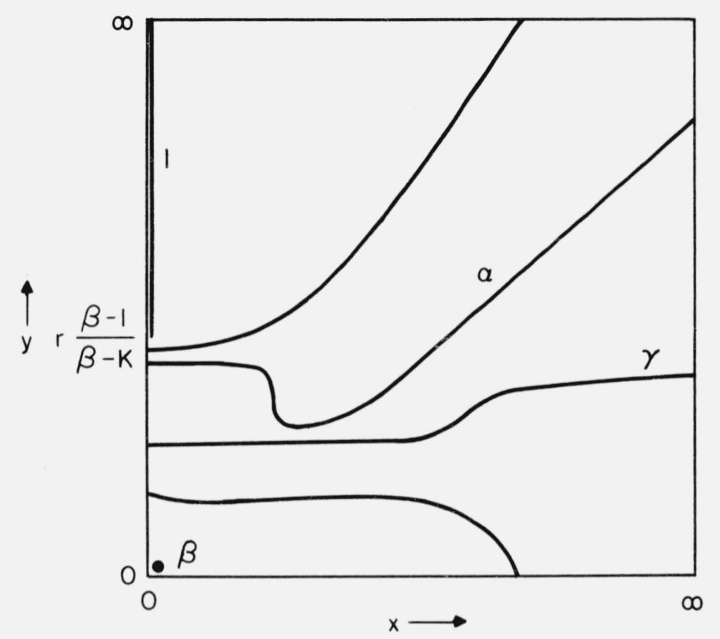

FigURE 11. Schematic contour diagram of index of refraction for mode III, on x-y plane.

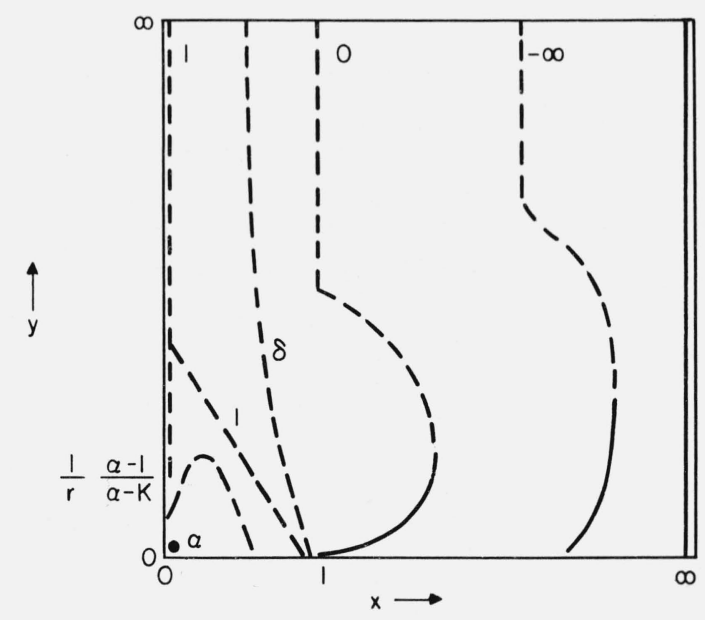

FiguRE 12. Schematic contour diagram of index of refraction for mode $I V$, on x-y plane. 


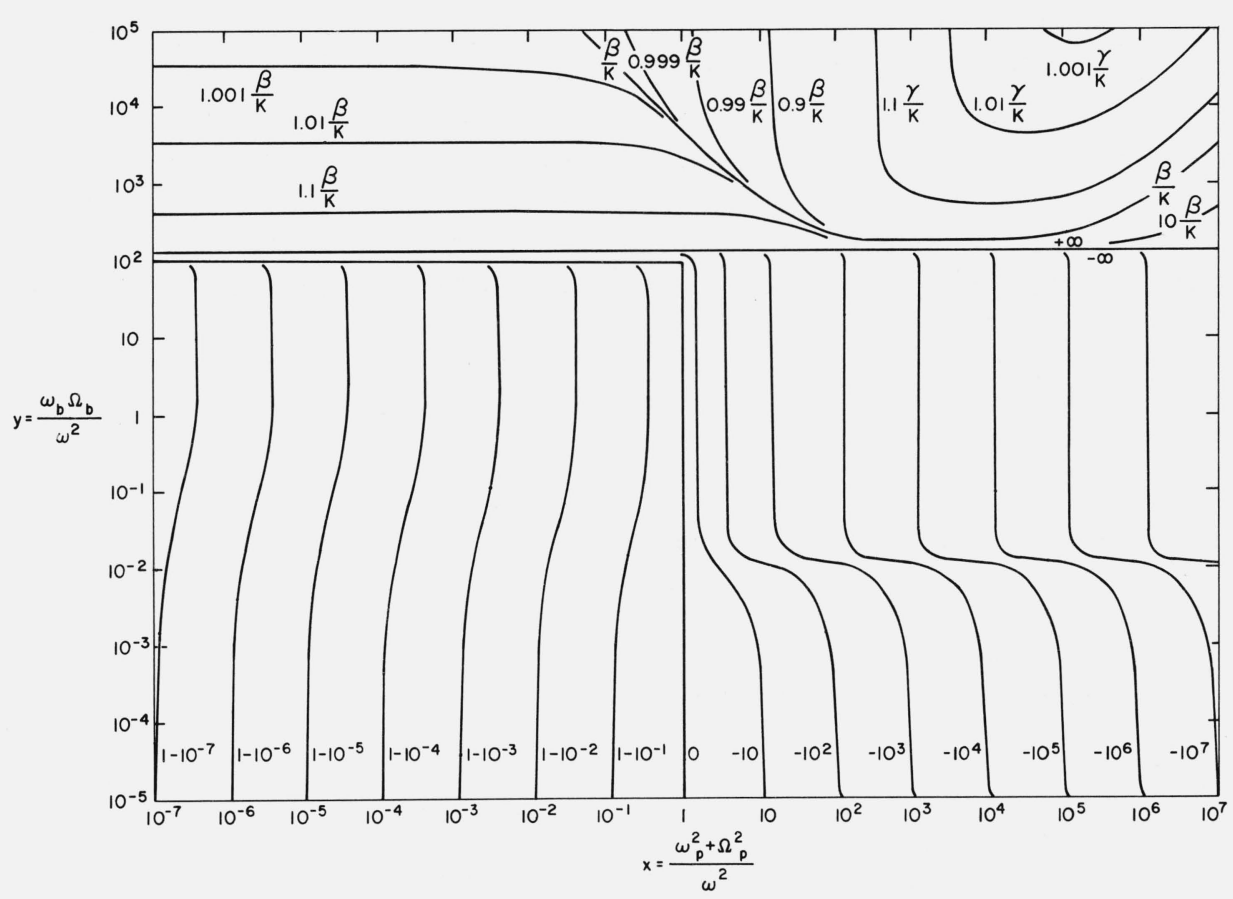

Figure 13. Contour diagram of index of refraction for mode $I$, for $\mathrm{r}=100, \mathrm{Z}=1, \alpha=50, \theta=30^{\circ}$.

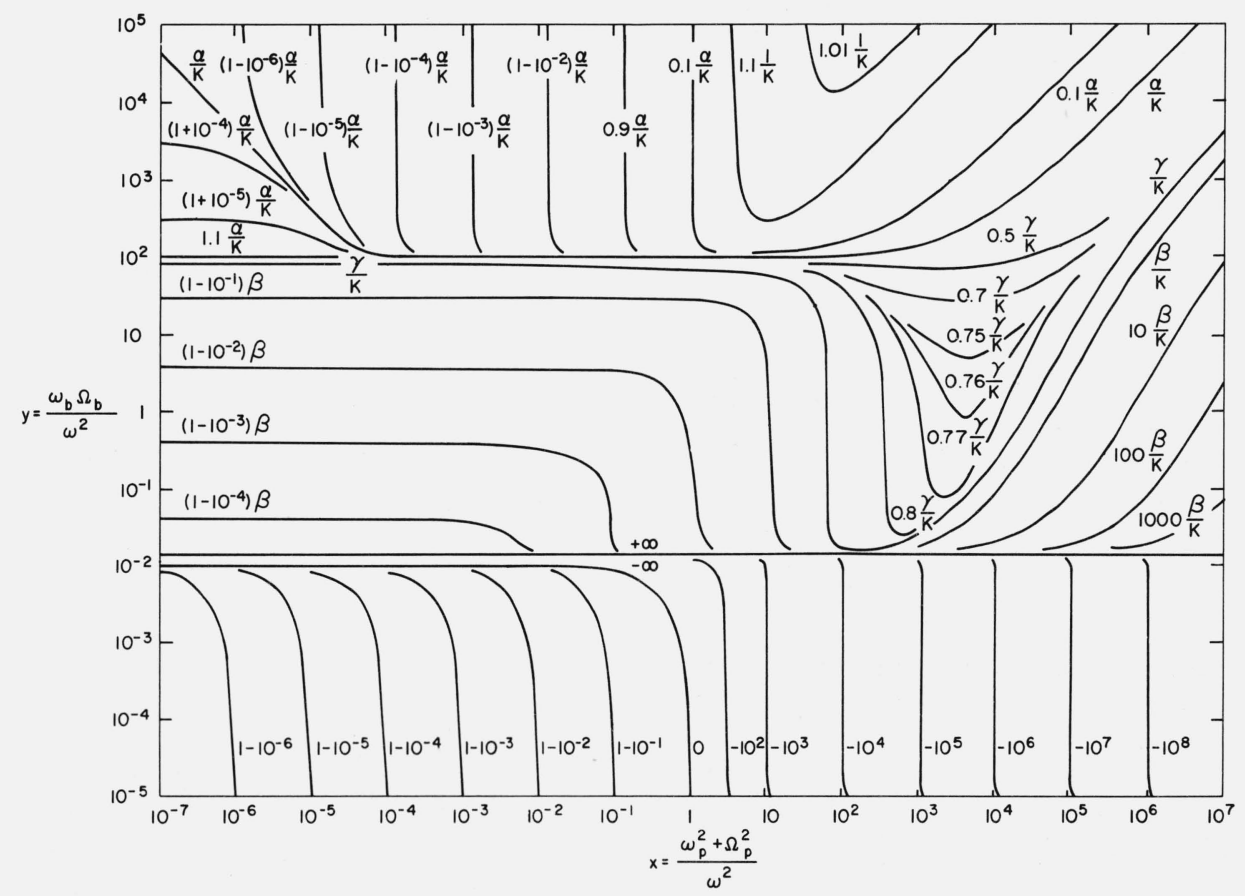

Figure 14. Contour diagram of index of refraction for mode II, for $\mathrm{r}=100, \mathrm{Z}=1, \alpha=50, \theta=30^{\circ}$. 


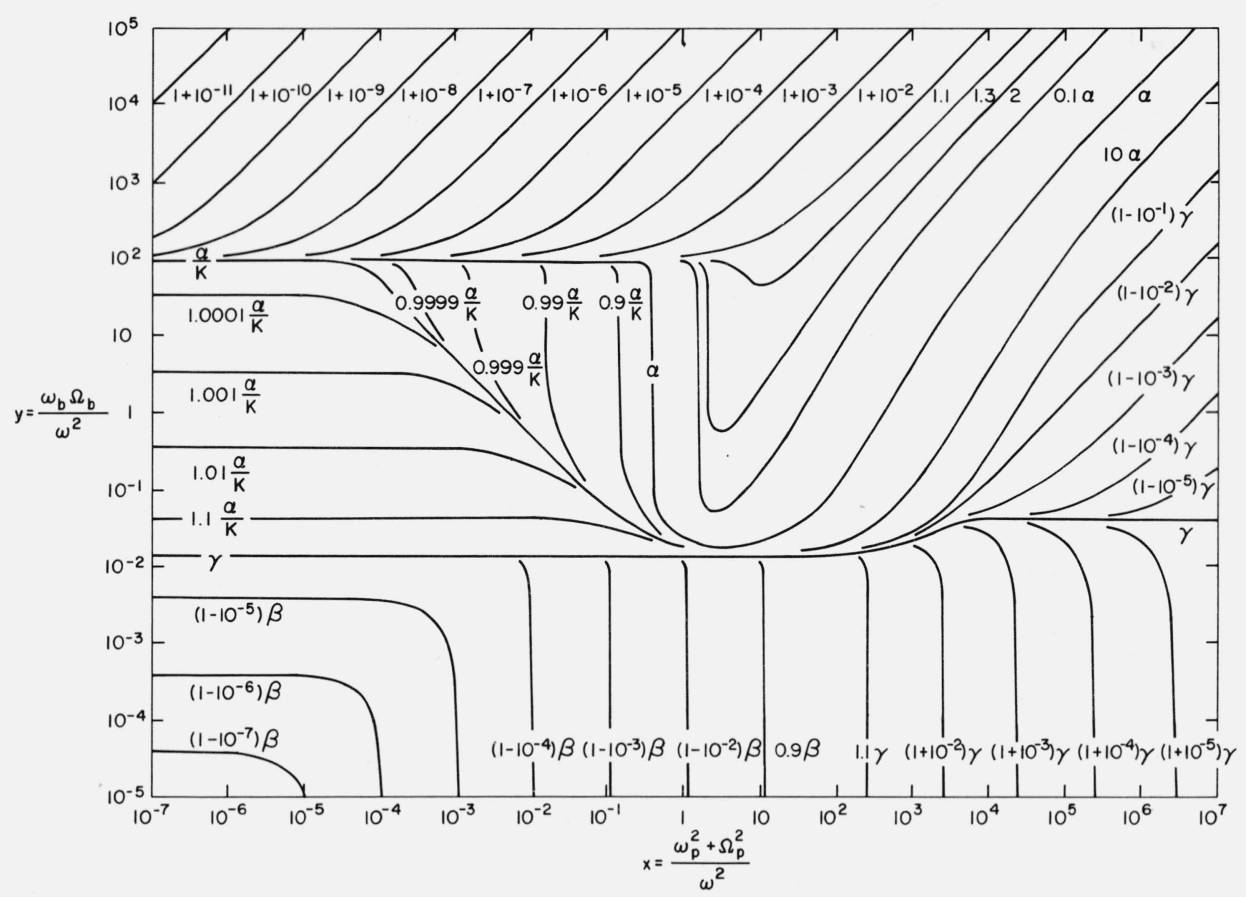

Figure 15. Contour diagram of index of refraction for mode III, for $\mathrm{r}=100, \mathrm{Z}=1, \alpha=50, \theta=30^{\circ}$. Curves are labeled with numerical value of $u=n^{2}$.

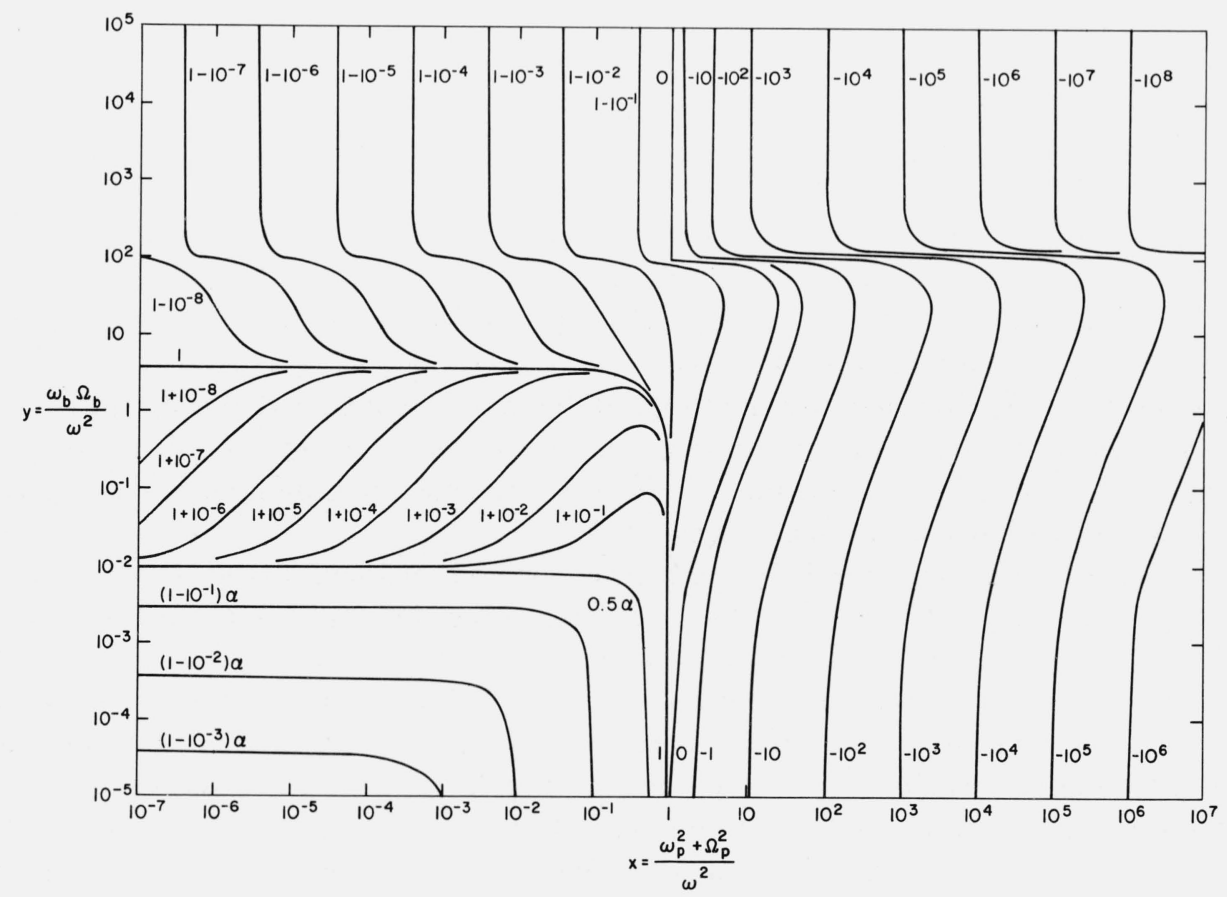

FIGURE 16. Contour diagram of index of refraction for mode $I V$, for $\mathrm{r}=100, \mathrm{Z}=1, \alpha=50, \theta=30^{\circ}$. Curves are labeled with numerical value of $u=n^{2}$. 
(1) Mode III has $u>0$ for all $x, y$, whereas modes I, II, IV have $u<0$ in some regions of the $x-y$ plane. These are regions where the corresponding waves are evanescent.

(2) Modes I, II, IV contain the line $u=0$, which is the boundary between propagation and evanescence. A point $u=0$ is called a cutoff.

(3) Modes I and II contain singularities, $u= \pm \infty$. A point $u=+\infty$ is called a resonance.

(4) Modes II, III, IV contain regions where the surface has a very steep slope, falling abruptly from $\gamma / K$ to $\alpha / K$ (mode II), from $\alpha / K$ to unity and from $\beta$ to $\alpha$ (mode III), and from $\alpha$ to unity (mode IV). These regions are called pseudo resonances. In the limit $T \rightarrow 0$ they turn into the true resonances of magnetoionic theory. [Denisse and Delcroix, 1961.]

(5) The regions of oblique asymptotes of modes I, II, and III are the regions of the three magnetohydrodynamic waves. Modes I and III give the slow and fast magnetoacoustic waves, while mode II gives the Alfvén wave. Equation (7) gives the Alfvén velocity, divided by $K$, as the wellknown asymptotic phase velocity of mode II.

(6) The valleys in modes II and III give minima in the dispersion curves. The valley of mode III is the region of common whistlers. The valley of mode II is a region of MHD whistlers. These MHD whistlers do not appear to have been discussed much in the literature, although the minimum in the mode II dispersion curve shows in figure VI, 10 of Denisse and Delcroix [1961]. As shown in figure 18 below, this minimum does not appear except at very small values of $y / x$.

(7) The cold plasma modes can be found by letting $\alpha, \beta, \gamma \rightarrow \infty$. The surfaces can then be coalesced into 2 sheets, the ordinary and extraordinary waves.

\section{Dispersion Curves}

Dispersion curves giving $u(\omega)$ are obtained by cutting the index of refraction surfaces by planes $y / x=\omega_{b} \Omega_{b} /\left(\omega_{p}^{2}+\Omega_{p}^{2}\right)$. Each plane is specified by a specific ratio of plasma density to strength of the static magnetic field. The dispersion curves for each mode are confined to limited regions on the $u$-x plane. This is displayed in figures 17 to 20 , each of which is the family of dispersion curves for one mode, for a wide range of values of $(y / x)$.

It is of interest to collect together the four dispersion curves for the various modes for constant $y / x$. This is done in figures 21 to 25 , which show the combined curves for $y / x=10^{4}, 10^{2}, 1,10^{-2}$, $10^{4}$.

The dispersion curves are constructed from the index surfaces, figures 13 to 16 , and thus apply to that particular plasma, although they are similar for other values of the parameters. Denisse and Delcroix [1961, See ch. VI] have given a set of dispersion curves comparable to figures 21 to 25 for the case $r=1836, Z=1, \alpha \approx 10^{5}, \theta=0^{\circ}$.

In ionospheric work it often is useful to regard the magnetic field as constant while letting the density vary. The dispersion curves corresponding to this situation are obtained by cutting the index surfaces with planes at constant $y$. The profiles are again plots of $u$ versus $x$, but they may be interpreted as plots of $u$ versus height.

In radio astronomy there often is interest in following a monochromatic ray through a region where $B$ and $N$ both vary. The set of values $(x, y)$ along the ray's trajectory describes a curve on the $x-y$ plane, and the curve can be drawn on each of the four index surfaces. The value and gradient of the index can thereby be seen for each of the four waves, and with this information one may be able to infer something of the nature of the source. [Pawsey and Bracewell, 1955] The simple assumptions concerning the propagation which one generally makes are (a) a wave is totally reflected at a cutoff; (b) a wave is totally absorbed at a resonance or at a pseudo-resonance; (c) a wave suffers high attenuation if its phase velocity is comparable with the thermal velocity of the plasma particles; and (d) energy can be coupled from one wave to another in regions of high gradient or in regions where two different modes have nearly the same index. (For theoretical discussions of these processes, see, e.g., Stix [1962], Budden [1961], and Ginzburg, [1961].) 


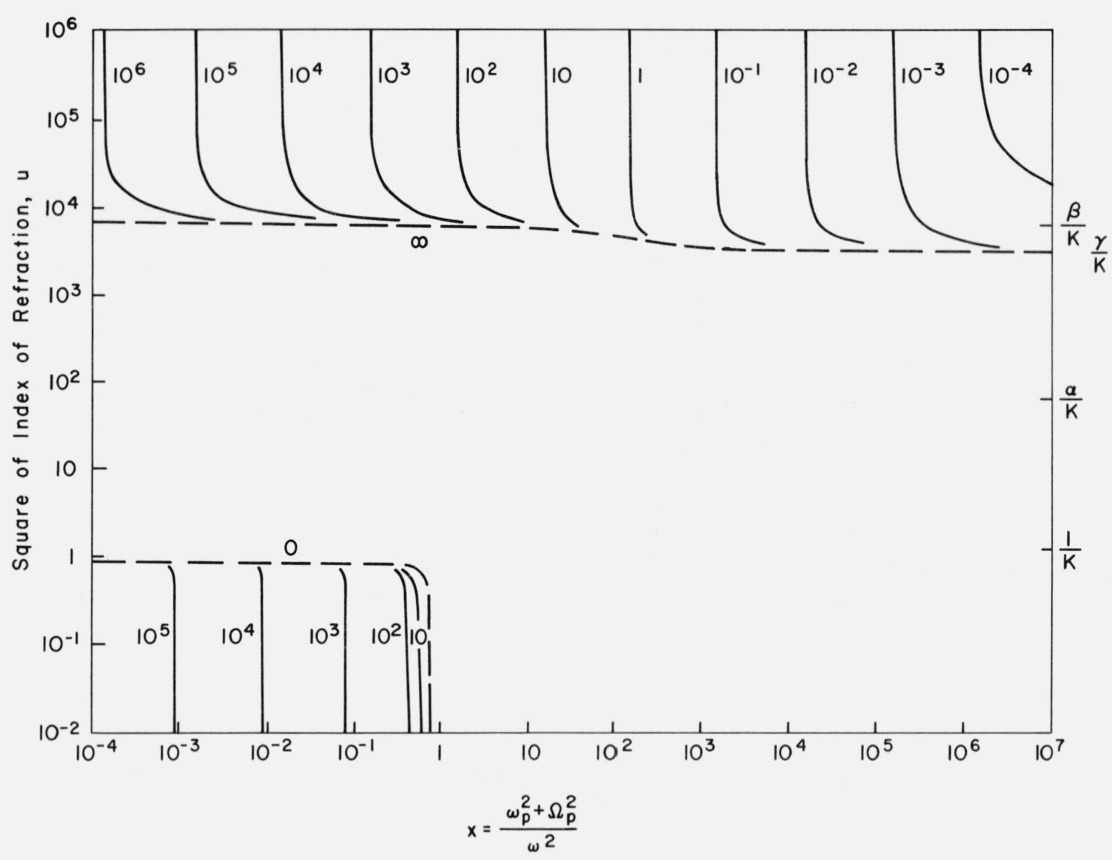

Figure 17. Dispersion curves for mode I, Parametric in $\mathrm{y} / \mathrm{x}=\omega_{\mathrm{b}} \Omega_{\mathrm{b}} /\left(\omega_{\mathrm{p}}^{2}+\Omega_{\mathrm{p}}^{2}\right)$, for $\mathrm{r}=100, \mathrm{Z}=1, \alpha=50$,
$\theta=30^{\circ}$

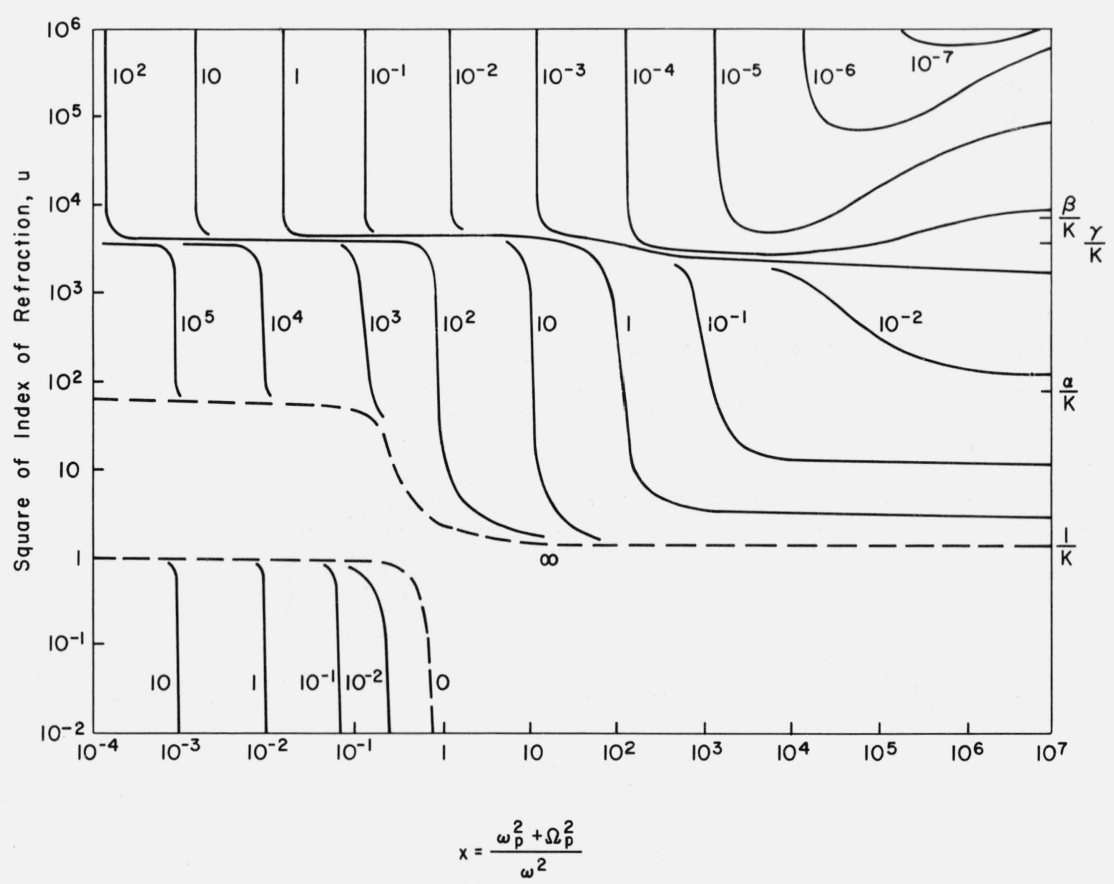

FigURE 18. Dispersion curves for mode 11 , parametric in $\mathrm{y} / \mathrm{x}=\omega_{\mathrm{b}} \Omega_{\mathrm{b}} /\left(\omega_{\mathrm{p}}^{2}+\Omega_{\mathrm{p}}^{2}\right)$, for $\mathrm{r}=100, \mathrm{Z}=1$,
$\alpha=50, \theta=30^{\circ}$. 


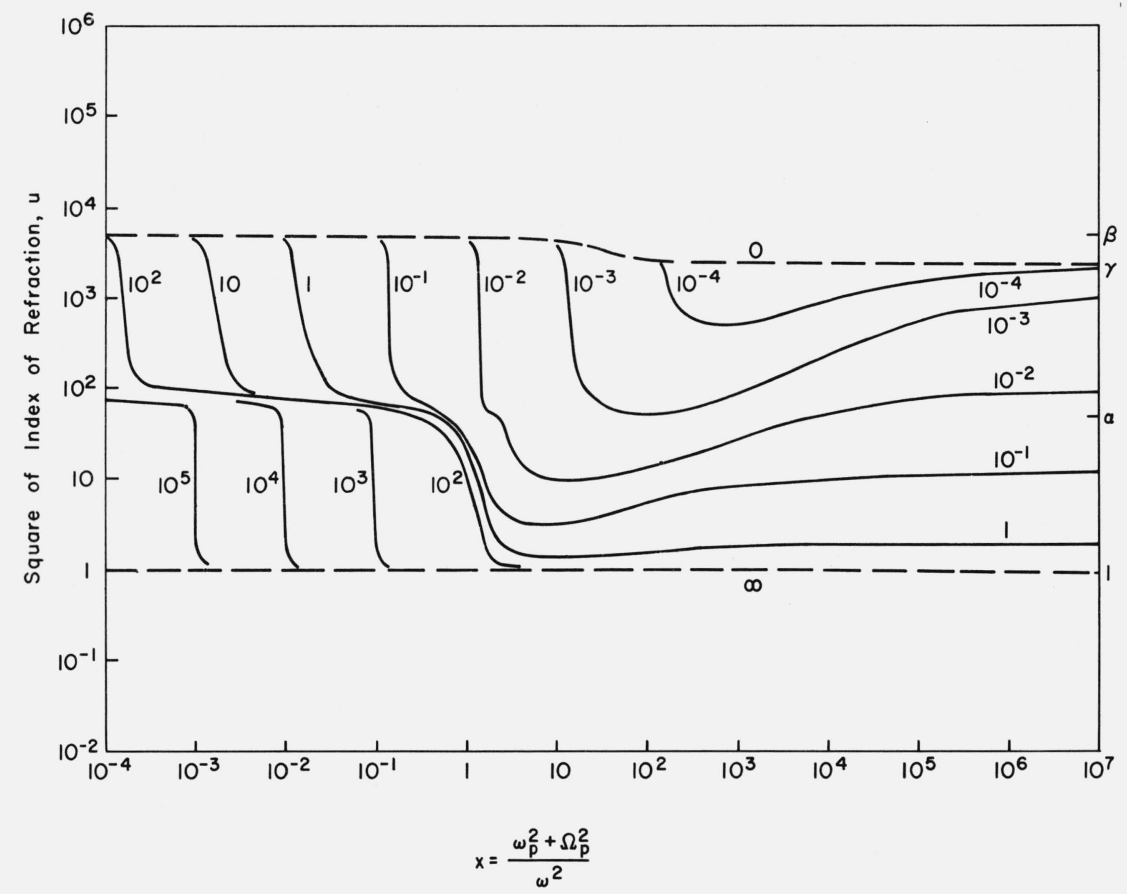

FIGURE 19. Dispersion curves for mode III, parametric in $\mathrm{y} / \mathrm{x}=\omega_{b} \Omega_{b} /\left(\omega_{\mathrm{p}}^{2}+\Omega_{\mathrm{p}}^{2}\right)$, for $\mathrm{r}=100, \mathrm{Z}=1$, $\alpha=50, \theta=30^{\circ}$.

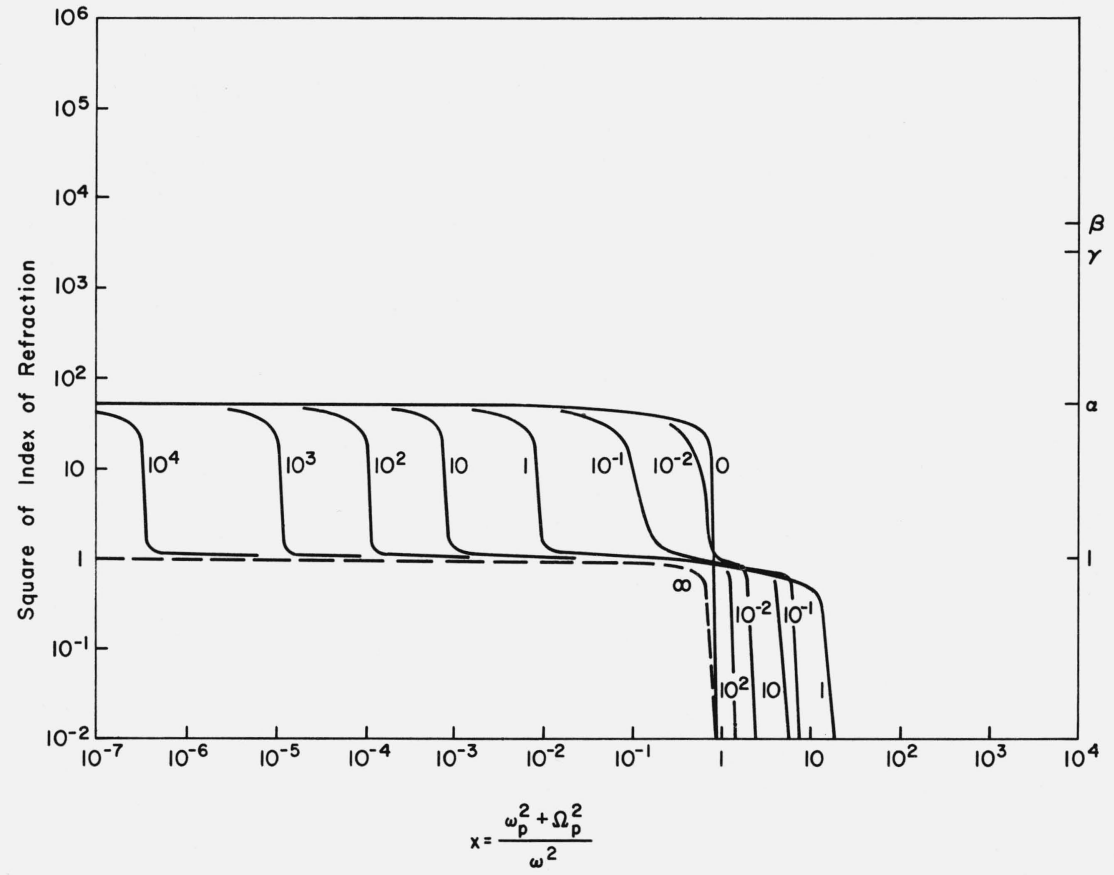

FiguRE 20. Dispersion curves for mode $I V$, parametric in $\mathrm{y} / \mathrm{x}=\omega_{\mathrm{b}} \Omega_{\mathrm{b}} /\left(\omega_{\mathrm{p}}^{2}+\Omega_{\mathrm{p}}^{2}\right)$, for $\mathrm{r}=100, Z=1$, $\alpha=50, \theta=30^{\circ}$. 


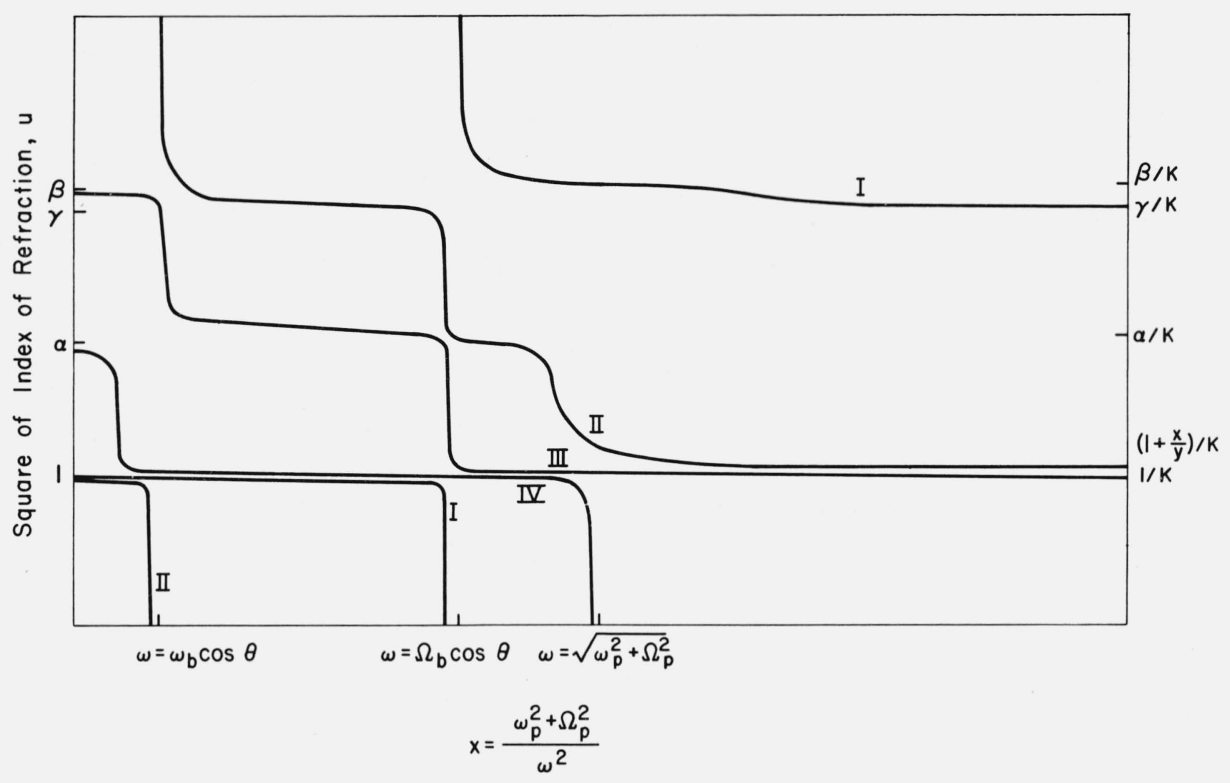

Figure 21. Dispersion curves for $\mathrm{y} / \mathrm{x}=\omega_{\mathrm{b}} \Omega_{\mathrm{b}} /\left(\omega_{\mathrm{p}}^{2}+\Omega_{\mathrm{p}}^{2}\right)=10^{4}$, for $\mathrm{r}=100, \mathrm{Z}=1, \alpha=50, \theta=30^{\circ}$.

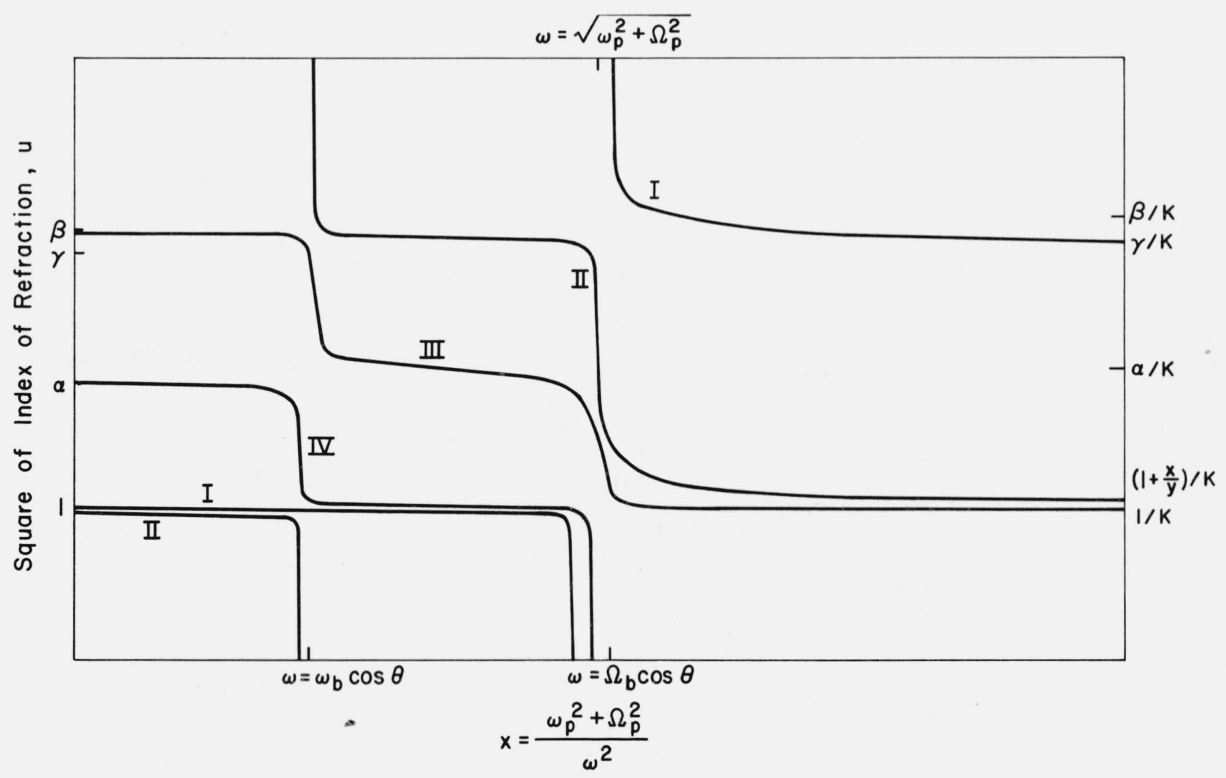

FIGURE 22. Dispersion curves for $\mathrm{y} / \mathrm{x}=\omega_{\mathrm{b}} \Omega_{\mathrm{b}} /\left(\omega_{\mathrm{p}}^{2}+\Omega_{\mathrm{p}}^{2}\right)=10^{2}$, for $\mathrm{r}=100, \mathrm{Z}=1, \alpha=50, \theta=30^{\circ}$. 


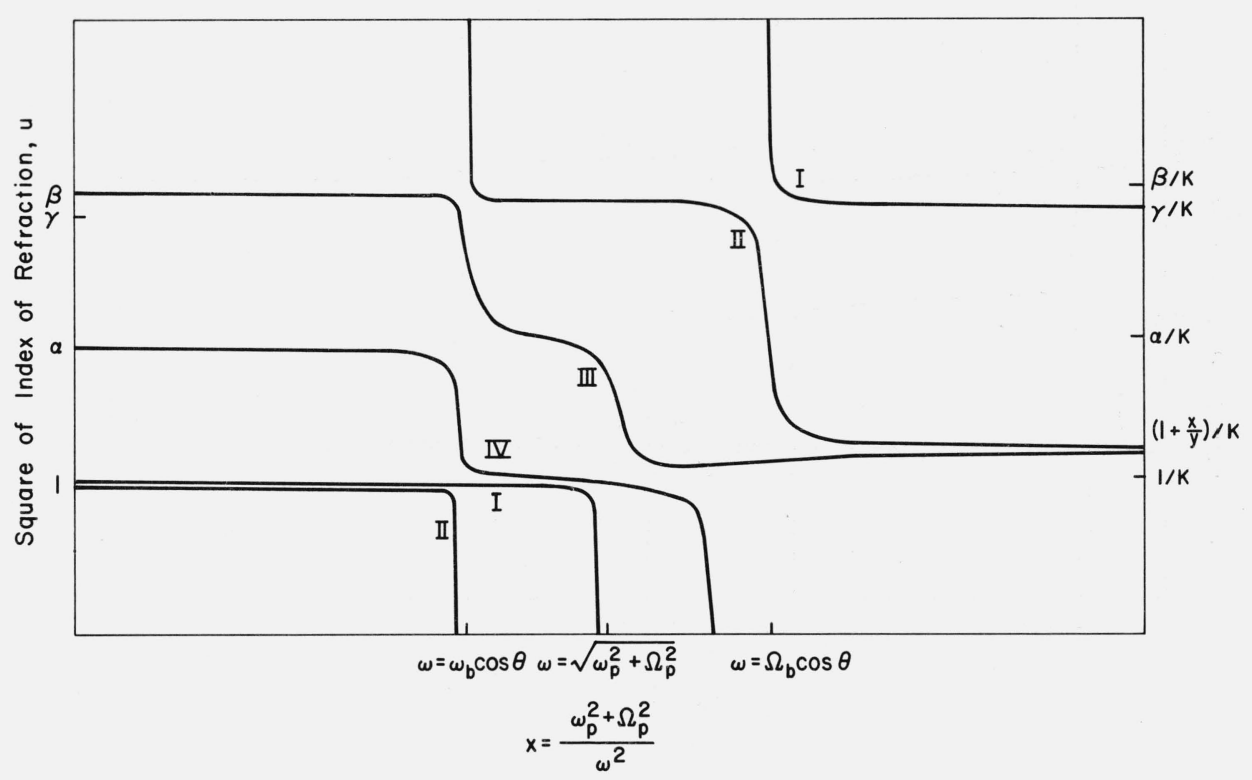

Figure 23. Dispersion curves for $\mathrm{y} / \mathrm{x}=\omega_{\mathrm{b}} \Omega_{\mathrm{b}} /\left(\omega_{\mathrm{p}}^{2}+\Omega_{\mathrm{p}}^{2}\right)=1$, for $\mathrm{r}=100, \mathrm{Z}=1, \alpha=50, \theta=30^{\circ}$.

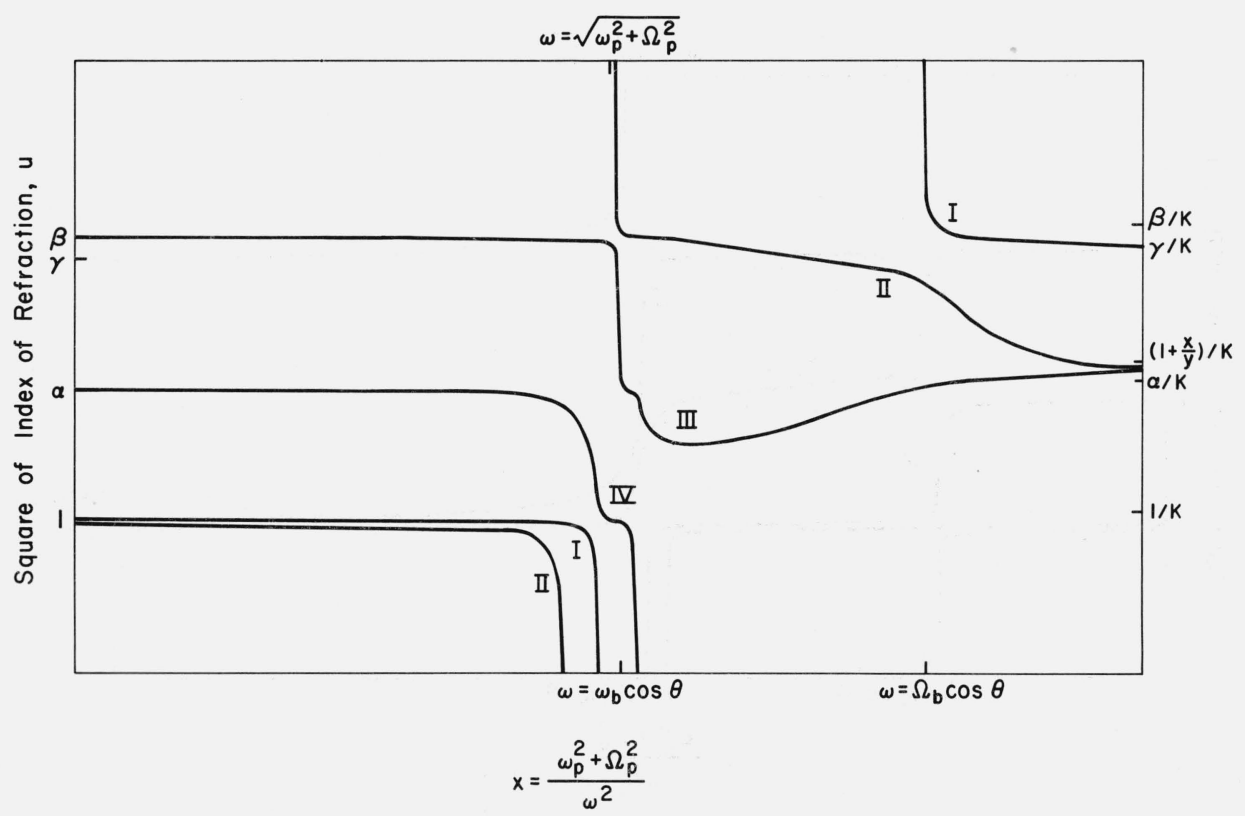

Figure 24. Dispersion curves for $\mathbf{y} / \mathbf{x}=\omega_{\mathrm{b}} \Omega_{\mathrm{b}} /\left(\omega_{\mathrm{p}}^{2}+\Omega_{\mathrm{p}}^{2}\right)=10^{-2}$, for $\mathrm{r}=100, \mathrm{Z}=1, \alpha=50, \theta=30^{\circ}$. 


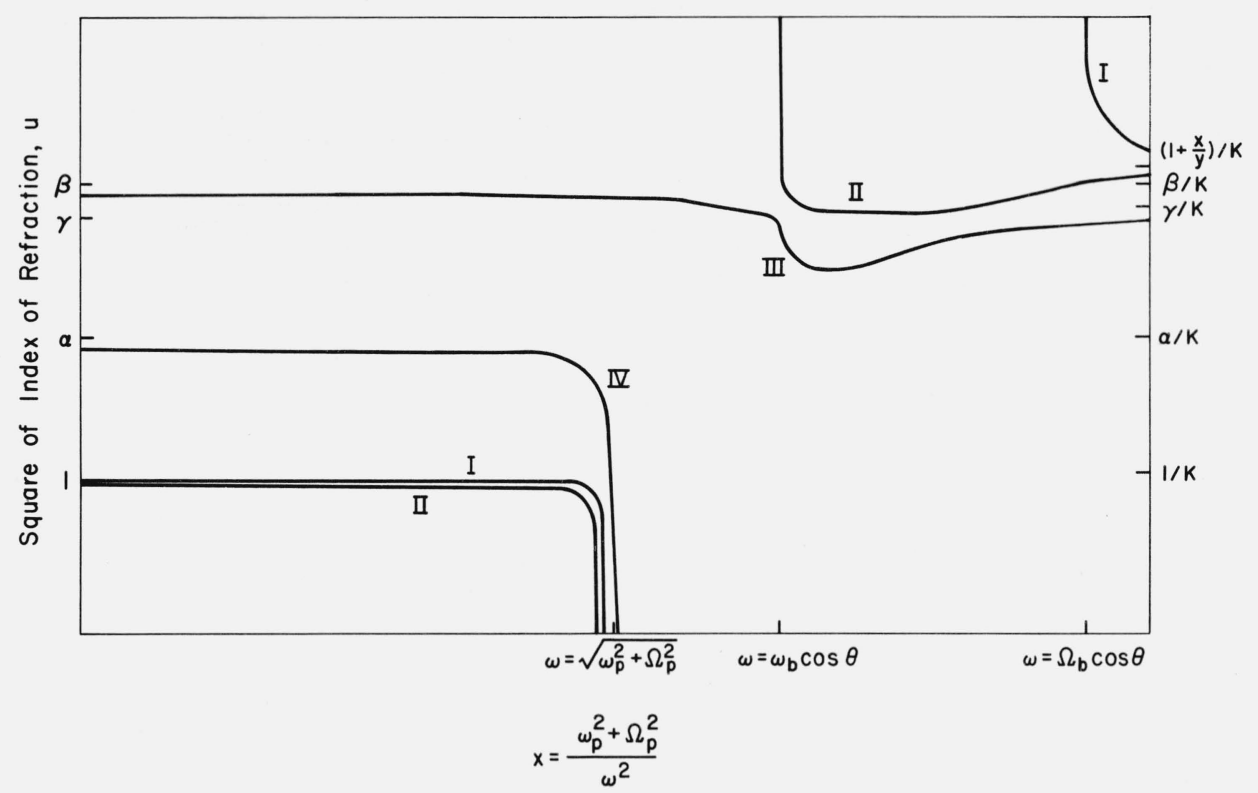

FIGURE 25. Dispersion curves for $\mathrm{y} / \mathrm{x}=\omega_{\mathrm{b}} \Omega_{\mathrm{b}} /\left(\omega_{\mathrm{p}}^{2}+\Omega_{\mathrm{p}}^{2}\right)=10^{-4}$, for $\mathrm{r}=100, \mathrm{Z}=1, \alpha=50, \theta=30^{\circ}$.

This work was partially supported by ARPA as part of Project Defender and was monitored technically by AFOSR, Contract AF-49 (638)-1156.

\section{References}

Allis, W. P., S. J. Buchsbaum, and A. Beers (1963), Waves in Anisotropic Plasmas (MIT Press, Cambridge, Mass.). Budden, K. G. (1961), Radio Waves in the Ionosphere (Cambridge University Press, Cambridge, England).

Denisse, J. F. and J. L. Delcroix (1961), Théorie des Ondes Dans les Plasmas, Ch. II and IV (Dunod, Paris, France).

Ginzburg, V. L. (1961), Propagation of Electromagnetic Waves in Plasma (Gordon and Breach, Inc., New York, N.Y.). Pawsey and R. N. Bracewell (1955), Radio Astronomy, p. 88 (Oxford University Press, London, England).

Stix, T. H. (1962), The Theory of Plasma Waves (McGraw-Hill Book Company, Inc., New York, N.Y.).

(Paper 69D4-490) 